\title{
Popular American plant names.
}

FANNIE D. BERGEN.

[At the request of the author and from plates kindly furnished by the editor of the Journal of American Folk-lore, Mr. W. W. Newell, the following is reprinted from that journal, both because of its intrinsic interest to botanists and for the sake of assisting the author in getting a more complete list of well authenticated local names. In this endeavor our readers are urged to coöperate, by sending such names to Mrs. Fannie D. Bergen, i 7 Arlington st., North Cambridge, Mass.-EDs.]

THE following list of names of common wild and cultivated plants has been prepared in the hope that it may suggest to folk-lorists who have some acquaintance with botany the importance of recording and communicating such names as may come to their knowledge. This work has been very thoroughly done in Great Britain; it is time that something like it should be attempted for our own flora.

In some cases, when I have taken the name from some one's description, there has been uncertainty as to the species, although there was no doubt about the genus; so that, in a few instances, I have only been able to give the latter.

It is interesting to notice the part certain nouns, used as adjectives or in composition, play in popular plant-names. Horse, cow, and bull have been generally used to designate unusually large and luxuriantly growing species, as the bull-thistle or horse-mint, or they are applied to coarse, common plants, as the horse-radish, the cowlily. Dog, pig, or sow generally seems to carry the idea of commonness, as dog-fennel, pig-weed, sow-thistle. Goose and toad are less frequently used in much the same sense, $e$. g. goose-grass, toad-flax. The word Indian we find in constant use to distinguish wild species from those tame or more familiar ones which they somewhat closely resemble. Mollugo verticillata is thus called Indian chickweed, to distinguish it from the omnipresent common chickweed, Stellaria media, which is naturalized from Europe. Not infrequently the "Indian" namesake of some well-known plant may be used as at least a nominal substitute for the latter, $e$. $g$. Indian tobacco, Antennaria plantaginifolia, is chewed by children. Now and then, hints and traditions of the use of certain plants in the rude medical practice of our Indians may have resulted in fastening the name Indian to that of these plants, and it is evident enough that the Indian rice, Zizania aquatica, owes the first part of its popular name to the great importance which some tribes attached to it as an article of food. 
The word snake plays an interesting part, too, in our popular botanical vocabulary. In general, "snake" indicates a plant supposed to be poisonous, or one which exerts a malign influence, yet sometimes it is applied to a plant that is thought to act as an antidote to the venom of snakes. A botanist from St. Stephen, N. B., writes : "Almost any unfamiliar berry is or may be snake-berry, and all snake-berries are poisonous; so a boy dares not eat a berry till some one tells him that it is good. Hence, though no two agree as touching the identity of the snake-berry, the name is very common." I find, too, curiously enough, that "snake" is sometimes used by a people no less widely removed from us than the Japanese to designate fruit unfit to be eaten by man. For instance, a beautiful large red fruit much resembling the strawberry, but whose flavor is perfectly insipid, is popularly called snake-berry, signifying that it is only fit food for snakes. Our popular name of Devil's apron for the familiar kelp, Laminaria longicnuris, doubtless arises from the giant size of some of these plants, and I am told that in Japan this prefix sometimes designates an unusually large species. For instance, a monstrous thistle is called devil-thistle. Also a large variety of the particular rhomboidal-shaped Chinese nuts called hishi are popularly known in Japan as devil-hishi. However, with the Japanese as with us, devil may mean " armed," or uncanny in appearance, as the "devil-lotus," one with very prickly leaves. Our wellknown prickly pear, Opuntia Rafinesqii or O. vulgaris, when cultivated in northern Ohio, is somewhat generally known as devil's tongue, which must seem a most fitting name to any one who has imprudently filled the tips of his fingers with the insinuating barbed bristles.

As a rule, I have here entered only such popular names of wild plants as are not recorded in the new edition of Gray's Manual. Wood's Botany contains some of those that I have collected from various parts of the country, but such as I have here retained as are found in either of these floras are given for the sake of designating special localities for such names, or because of some note that seemed worth appending.

In those instances in which I have given as locality only the name of the State, it is either because the name is known to be in use in various parts of the State, or because my informant could not give the county or town. Some names given are such as were certainly current a good many years ago in the localities cited, but have not been verified as still existent there. It would often have been very difficult to make inquiries about the present currency of these names ; hence they have been allowed to stand as probably still in use. 
RANUNCULACEA.

Clematis Virginiana, traveller's joy ; wild hops. N. H. devil's darning needle. So. Vt.

Anemone nemorosa, wild cucumber. N. H.

Mayflower. Boston.

Hepatica triloba, mouse-ears. Mason, N. H.

Mayflower. Hemmingford, P. Q.

Anemonella thalictroides, wind-flower. Mansfield, O.

Thalictrum polygamum, rattlesnake-bite. N. H.

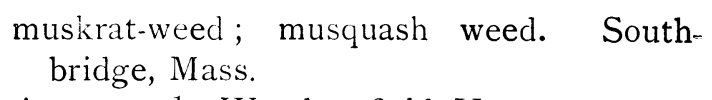

Thalictrum dioicum, shining grass. ${ }^{1}$ Weathersfield, Vt.

Ranunculus (double garden buttercups), golden claisies. Richland Co., O.

Ranunculus aquatilis, var. trichophyllus, moss (gives name to "Moss Creek," Carroll Co., Mo.).

Caltha palustris, May-blobs. Salem, Mass.

coltsfoot. Stratham, N. H.

Coptis trifolia, yellow-root. N. H.

Nigella Damascena, love-in-a-mist; lady-in-the-green. N. E. and

Westward.

lady-in-a-chaise. N. H.

devil-in-a-bush. Northern Ohio.

St. Catherine's flower. (Locality ?)

ragged lady. Wisconsin.

Aquilegia Canadensis, honeysuckle. N. E. ; Peoria, Ill.

rock-lily. Mason, N. H.

cluckies. Annapolis Co., N. S.

meeting-houses. New England.

Aconitum Napellus, Venus' chariot. ${ }^{2}$ Brookline, Mass. Acte spicata, var. rubra, snake-berry. Belleisle, N. B.

\section{NYMPHÆACEÆ.}

Nelumbium luteum, chinquapins. Carroll Co., Mo.

Nuphar advena, cow-lily. Washington Co., Me.

dog-lily. New England.

beaver-lily. Me.

bull-head lily. N. H.

ducks. $^{3} \quad$ Chestertown, $\mathrm{Md}$.

1 See, also, Impatiens. The name is given because of the silvery appearance of the leaves when immersed in water.

2 The swans are hidden in the hood.

${ }^{8}$ Quy. docks, as in spatter-dock? 


\section{SARRACENIACE $Æ$.}

Sarracenia purpurea, Adam's cup. Dudley, Mass. foxglove. N. H.

Indian pitcher. N. B.

PAPAVERACE $E$.

Eschscholtzia, California poppy. General. cups-of-flame. New England.

Papaver (a small species), coquettes. ${ }^{1}$ Mansfield, O. Argemone Mexicana, bird-in-the-bush. Arlington, Mass. flowering thistle. Mansfield, O.

Sanguinaria Canadensis, snake-bite. N. H.

\section{FUMARIACEÆ.}

Adlumia cirrhosa, Alleghany vine. N. Ohio. mountain fringe. So. Vt.; E. Mass. fairy creeper. Fredericton, N. B.

Dicentra spectabilis, diethra. Mass.

\section{CRUCIFER E.}

Lepidium Virginicum, birds' pepper. Nebraska.

Capsella bursa-pastoris, pepper-plant. Allston, Mass.

\section{VIOLACE $Æ$.}

Viola palmata, var. cucullata, hood-leaf violet. Franklin, Mass.

Viola (sp. unknown), rooster hoods. Buncombe Co., N. C.

Viola sagittata, spade-leaf violet. Franklin, Mass.

Viola Canadensis, June flower. Woodstock, N. B. ; Houlton, Me.

Viola tricolor, lady's delight. Mass.

Cupid's delight. Salem, Mass.

Johnny-jump-up. ${ }^{2}$ O. and Ill.

Viola pedata, horseshoe violet. Concord, Mass.

Crowfoot violet. New England.

horse violet. New England.

DROSERACE A.

Drosera rotundifolia, eye-bright. N. H.

\section{CARYOPHYLLACEE.}

Dianthus barbatus, bunch pink. Vt.; So. Ohio.

Saponaria officinalis, old maid's pink; London pride. Salem, Mass. woods phlox. N. J.

1 French coquelicot.

2 In Mansfield, Ohio, this name is commonly abbreviated into Johnnies, and this nickname is often applied by children to the common wild blue violet. 
Silene cucubalus, snappers. Salem, Mass.

Silene Armeria, wax-plant. Mansfield, O.

sweet Susan. N. H.

none-so-pretty. Hatfield, Mass.

pretty Nancy. Franklin Center, P. Q.

Silene noctiftora, gentlemen's hats. Gilsum, N. H.

Lychnis Githago, old maid's pink. N. H.

mullein pink. Annapolis Valley, N. S.

Lychnis chalcedonica, sweetwilliam. Weathersfield, Vt.; So. Ohio.

fire-balls. Mansfield, O.

scarlet lightning. ${ }^{1}$ Hemmingford, P. Q.

PORTULACACEE.

Portulaca oleracea, pusley. U. S.

Portulaca grandiflora, Mexican rose. Chestertown, Md.

rose-moss. So. Nebraska.

French pusley. So. Vt.

Claytonia Virginica, good-morning-spring. (Locality ?)

wild potatoes. Union Co., $\mathrm{Pa}$.

Mayflower. Hemmingford, P. Q.

\section{MALVACE $Æ$}

Abutilon Avicenne, butter-weed. Peoria, Ill.

sheep-weed; Mormon-weed ; velvet-weed.

Quincy, Ill.

button-weed. Chestertown, Md.

Abutilon striatum, flowering maple. Mansfield, O.

Malva rotundifolia, cheeses, or cheese-plant. U. S.

Malva moschata, musk-plant or musk. Mansfield, O.

Hibiscus trionum, black-eyed Susan. N. H. ; N. B.

devil's-head-in-a-bush. N. H.

GERANIACEA.

Geranium maculatum, chocolate-flower. Stratham, N. H.

Pelargonium (common pink and white species or var.), apple gera. nium. Mansfield, O., and parts of Mass.

Oxalis stricta, ladies' sorrel. Allston, Mass. ; Stratham, N. H.

Impatiens fulva, snap-dragon. N. H.

snap-weed. N. B.

kicking colt. E. Mass.

shining grass. ${ }^{2}$ Weathersfield, Vt.

1 Probably a corruption for Lychnis.

2 See note on Thalictrum dioicum. 
balsam-weed ; slipper-weed ; lady's ear-drop. Mansfield, $\mathrm{O}$.

lady's slipper. Plattsburg, N. Y.; Mansfield, O

lady's pocket. Mansfield, $\mathrm{O}$.

Impatiens balsamina, lady's slipper. Mansfield, $O$.

ILICINEF.

Nemopanthes fascicularis, brick-timber ; cat-berry. ${ }^{1}$ Fortune Bay, Newfoundland.

\section{CELASTRACEF.}

Celastrus scandens, Roxbury wax-work. E. Mass.

Jacob's ladder. Stratham, N. H.

Euonymus atropurpureus, Indian arrow. Salem, Ind.

Pachystima Canbyi, rat-stripper. N. J.

\section{VITACE $\nexists$.}

Vitis cordifolia, chicken grapes. Chestertown, Md.

ANACARDIACE.E.

Rhus glabra, shoe-make. Ohio and Ill.

Rhus toxicodendron, black mercury. Harmony, Me.

mercury or markry. N. H.

mark-weed. Kennebec Co., Me.

POLYGALACE $\mathbb{E}$.

Polygala paucifolia, babies' feet. N. H.

babies' toes. Hubbardston, Mass.

\section{LEGUMINOSA.}

Crotalaria (ovalis?), rattlesnake-weed. Mansfield, O.

Genista tinctoria, wood-wax. Essex Co., Mass. ${ }^{2}$

Lupinus perennis, wild pea. Worcester Co., Mass.

Lupimus villosus, monkey faces; sun-dial. ${ }^{3} \mathrm{~N}$. Ohio.

Trifolium pratense, "real sweet clover." Mass. and parts of Me.

Amorpha canescens, shoestrings. Ill.

Apios tuberosa, traveller's delight. New Albany, Miss.

wild bean. N. B.

Phaseolus multiflorus, flower bean. Mansfield, O.

1 This, like most of the other names quoted from Newfoundland, is taken from Rev. A. C. Waghorne's Wild Berries and other Edible Fruits of Nerufoundland and Labrador.

2 In this its principal American locality, the plant is never called wood-waxen, or any other name than that above given.

8 So called from the monkey-like profile of the seed. 
Arachis hypogaa, ground-nut. Chestertown, Md.

goobers. Southern.

pinders. Miss.

ground-peas. $\mathrm{Ky}$.

Schrankia uncinata, sensitive rose. West and South.

Schrankia sp., shame-vine. N. Miss.

ROSACEA.

Prunus serotina, rum-cherry. ${ }^{1} \quad$ N. E.

Prunus Americana, wild goose plum. Chestertown, Md.

Prunus hortulana, wild goose plum. Markets of Boston and else. where.

Prunus maritima (?), mountain cherry. Chestertown, Md.

Spircea sp., spice hard-hack. Bonny River, N. B.

Rubus odoratus, mulberry ; Scotch caps. Hemmingford, P. Q.

Rubus chamamorus, baked apples. New Brunswick and Grand

Manan Id.

bake-apple-berry. Grand Manan.

Rubus triflorus, mulberry. Washington Co., Me.; N. B.

dewberry. N. B.

plumbog. Newfoundland.

swamp-berry. Newfoundland.

Rosa cinnamomea, kitchen rose. Boston, Mass.

Pyrus arbutifolia, log-berry. N. E.

choke-pear. Washington Co., Me.

Pyrus Americana, witch-wood. ${ }^{2}$ N. H.

round-tree (for rowan-tree). N. B.

dog-berry. Newfoundland.

missey moosey. N. H.

Cydonia Faponica, scarlet thorn. Chestertown, $\mathrm{Md}$.

flowering quince. O., and somewhat general.

Crategus, thorn-apple. Mansfield, O.

Amelanchier Canadensis, June berry. Various parts of N. E. and Central States.

sugar plum; shad-blow. N. H.

sugar pear. Washington Co., Me.

juice-pear or juicy pear. Provincetown, Mass.

May-pear. ${ }^{3} \quad$ N. B.

1 From its use in flavoring "cherry rum." In the W. and S. whiskey is used with these cherries to make "cherry bounce."

2 If carried, supposed to keep off witches.

3 From time of blooming. 
SAXIFRAGACE $Æ$.

Saxifraga Virginiensis, Mayflower. Allston, Mass.

Ribes prostratum, skunk currant. ${ }^{1}$ Washington Co., Me.

Ribes aureum, flowering currant. General.

clove currant. Cambridge, Mass.

\section{CRASSULACE $Æ$.}

Sedum acre, love entangled. N. Ohio.

Sedum (pulchellum ?), flowering moss. Mansfield, O.

Sedum Telephium, witches' money-bags. W. Mass.

evergreen. Chestertown, Md.

everlasting. Hemmingford, P. Q.

Aaron's rod. New Hampshire.

frog's mouth ; frog's bladder. N. Y.

pudding-bag plant. Mass.

leeks. Stowe, Vt.

frog-plant. ${ }^{2}$ N. H.

frogs' throats. Bedford, Mass.

Sempervivum tectorum, hen and chickens. N. Ohio.

Bryophyllum calycinum, life-plant. Cambridge, Mass.

ONAGRACE $Æ$.

Enothera fruticosa, scabbish. N. H.

CUCURBITACEÆ.

Lagenaria sp., mock orange. N. Ohio ; Central Ill.

Echinocystis lobata, wild cucumber. N. B., and U. S. generally.

BEGONIACEÆ.

Begonia metallica, elephant's ears. Bedford, Mass.

Begonia maculata, trout begonia. Bedford, Mass.

fish begonia. Cambridge, Mass.

Begonia Warscewiczii, pond-lily begonia. Cambridge, Mass.

Begonia sp. (similar to B. maculata, but not spotted), coral begonia.

Bedford, Mass.

Begonia sp., beefsteak geranium. Mansfield, O.

strawberry geranium. Mansfield, $\mathrm{O}$.

\section{CACTACEÆ.}

Opuntia Rafinesqii, or
O. vulgaris.

1 From the offensive musky smell of the fruit.

2 Because of a children's custom of blowing up a leaf so as to make the epidermis puff up like a frog. 
FICOIDEÆ.

Mesembryanthemum sp., dew plant. N. Ohio.

rat-tail pink. Dorchester, Mass.

UMBELLIFERÆ.

Daucus carota, parsnip. Harmony, Me.

Erigenia bulbosa, turkey-pea. · (Locality?)

ARALIACE $Æ$.

Aralia racemosa, Indian root; life of man; petty morrell. N. H. spignut. $\mathrm{Vt}$.

CORNACE $Æ$.

Cornus Canadensis, bunch plums ; pudding-berry. ${ }^{1}$ N. H.

pigeon-berry. N. B.

cracker-berry. ${ }^{1} \quad$ Newfoundland.

Cornus stolonifera, red-brush. Central States.

Nyssa sylvatica, hornbeam. N. H.

CAPRIFOLIACEÆ.

Viburnum lantanoides, moosewood. Mass.

Viburnum opulus, high-bush cranberry. Washington Co., Me., and N. B.

witch-hobble. N. H.

Viburnum nudum, withe-wood. N. H.

bilberry. Annapolis Royal, N. S.

Linnae borealis, two-eyed berries. St. Stcphen, N. B.

Symphoricarpus racemosus, snow-drop. Manșfield, O.

RUBIACEÆ.

Houstonia corulea, blue-eyed babies. Springfield, Mass.

Quaker ladies. Concord, Mass. ;

Boston.

innocence. Boston, Mass.

eyebright. Isles of Shoals.

angel-eyes. (Locality?)

bright-eye. Baltimore, Md.

forget-me-not. Kentucky.

star of Bethlehem. Miss.

Quaker beauty. (Locality?)

Cephalanthus occidentalis, pin-ball. N. H.

Nuns. (Locality?)

1 Probably from its insipid character. 
Mitchella repens, squaw-vine. Parts of N. E.

snake-berry. N. Y.

cow-berry. Ulster Co., N. Y.

boxberry. Bedford, Mass.

two-eye-berry. Wakefield, Mass.

\section{COMPOSITE.}

Eupatorium purpureum, motherwort. Brookfield, Mass.

Queen-of-the-meadow. Worcester Co., Mass. marsh milkweed. Mass.

Solidago (any sp.), yellow-tops. N. B.

Callistephus Chinensis, fall roses. Mansfield, Ohio.

Aster (any sp.), frost-flowers. N. B.

Erigeron Canadense, cow-tail. Normal, Ill.

Antennaria plantaginifolia, Indian tobacco. N. E.; Neb.

woman's tobacco. Boston, Mass.

ladies chewing tobacco. Wisconsin.

pussy's toes. Worcester, Mass.

dog toes. N. H.

Anaphalis margaritacea (?), life-of-man. N. H.

Gnaphalium polycephalum, life everlasting. N. E. ; No. Ohio.

old field balsam. N. E.

life-of-man. Stratham, N. H.

fuzzy-guzzy. Mansfield, O.

feather-weed. ${ }^{1}$ No. New York.

Ambrosia artemisiafolia, tassel-weed. Hingham, Mass.

Zinnia elegans, youth-and-old-age. Mansfield, O.

Rudbeckia hirta, yellow daisies. Mass., N. B., and general.

golden Jerusalem. N. H. (local).

black-eyed Susans. N. Vt.; Cape Cod.

nigger-heads. (Name apparently brought from So. U. S.) N. B.

nigger daisy. E. Mass.

Coreopsis tinctoria, Rocky Mt. flower. Mansfield, O.

Bidens (all species), Spanish needles. Ill., and Central States generally.

Anthemis cotula, dog-fennel. General.

pigsty daisy. Ipswich, Mass.

Chrysanthemum leucanthemum, pismire. East Weymouth, Mass. bullseye. N. B.

1 Name given because the heads were used by poor people to fill beds, as a substitute for feathers. 
Artemisia abrotanum, boy's love $;^{1}$ lad's love. ${ }^{1}$ Various parts of New England. old man. ${ }^{1}$ Ohio ; Ill. Leamington. Ipswich, Mass.

Artemisia sp., old woman. ${ }^{1} \quad$ N. Ohio.

Xeranthemum,

Helichrysum, paper-flowers. N. Ohio.

Cnicus pumilus, bull-thistle. New England.

Cnicus (any species), stickers. St. John, N. B.

Cichorium Intybus, blue dandelion. N. H.

$$
\text { blue sailors. Brooklyn, N. Y. }
$$

Leontodon autumnalis, arnica. E. Mass.

Lactuca (any species), milkweed. N. B.

\section{LOBELIACEA.}

Lobelia cardinalis, slink-weed. Princeton, Mass.

\section{ERICACE $\not$.}

Gaylussacia (all species), black hurts. ${ }^{2}$ Newfoundland.

Vaccinium (many species), whortleberry; bilberry. Newfoundland. any low blueberry; ground-hurts. Newfoundland.

Vaccinium (any species under sub-genus Cyanococcus), bluets. N. B., among French Canadians.

Vaccinium Oxycoccus, marsh cranberry. N. B.

marshberry. Newfoundland.

Vaccinium macrocarpon, marsh cranberry. N. B.

bearberry; bankberry. Fortune Bay, Newfoundland.

Vaccinium Vitis-Idaa, rock cranberry. N. B.

Chiogenes serpyllifolia, ivory plums. Washington Co., Me.

capillaire; maiden-hair ${ }^{3}$ teaberry. New Brunswick.

Arctostaphylos uva-ursi, crowberry. Barnstable, Mass. ; Kinnikinnik,

Newfoundland.

rockberry. Fortune Bay, Newfoundland.

Epigcea repens, shad-flower. Conn.

1 Names apparently given from supposed aphrodisiac qualities, or because used in love divinations.

2 "Hurts" is an abbreviation for "whortleberry."

3 This name, attached to a description of the plant, was the occasion of an indignant protest by a botanist in England at the idea of the maidenhair (fern) being supposed to flower and fruit in New Brunswick ! 
Gaultheria procumbens, young plantlets ; drunkards. ${ }^{1}$ Barnstable, Mass.

youngsters. $^{2}$ Me.; Mass.

jinks or chinks. N. H. ; Mass.

young chinks. Mason, N. H.

pippins. $^{3}$ Stratham, N. H. ; Central Mass.

young ivories; ivory plums. N. H.

ivory leaves; ivory plums. Ipswich, Mass. ; Me.

mountain tea. Eastern Ohio.

ivy-berry. N. B.

deer-berry. (Locality ?)

one-berry. (Locality?)

chicken-berry. Penn.

Kalmia latifolia, spoon-hunt. Mason, N. H.

Kalmia angustifolia, sheep-poison. N. E.

spoon-wood ivy. Conn.

Rhododendron viscosum, swamp-pink. Allston, Mass.

Rhododendron nudiflorum, election pink. Hillsborough, N. H. river pink. Cavendish, Vt.

swamp pink. Parts of N. E.

swamp apple. E. Mass.

honeysuckle. Md.

Rhododendron Rhodora, lambkill. N. B.

Chimaphila umbellata, noble pine; bittersweet. N. H.

love-in-winter. Maine.

Chimaphila maculata, ratsbane; wild arsenic. Blue Ridge, Va.

Monotropa uniflora, convulsion-root. N. H.

ghost-flower. N. B.

DIAPENSIACEA.

Pyxidanthera barbulata, pyxie moss. N. J.

PRIMULACEÆ.

Primula grandiflora, polyanthus. So. Vt. ; Cambridge, Mass.; Mansfield, $\mathrm{O}$.

cups-and-saucers. Mansfield, O.

Trientalis Americana, Star-of-Bethlehem. N. H.

star anemone. Cambridge.

APOCYNACEE.

Vinca minor, myrtle. General.

1 Believed by children to intoxicate.

2 Young berries and shoots.

3 Young leaves. 
ASCLEPIADACEA.

Asclepias tuberosa, white root; yellow milk-weed. W. Mass.

\section{GENTIANACEÆ.}

Gentiana Andrezusii, blind gentian. Haverhill, Mass.

POLEMONIACE $Æ$.

Phlox pilosa, sweetwilliam. Fort Worth, Tex.

Phlox subulata, flowering moss. No. Ohio.

Phlox, cult. sp., Lady Washington. Mansfield, O.

Polemonium reptans, bluebell. Mansfield, O.

BORRAGINACE $A$.

Cynoglossum officinale, sheep-lice. No. Ohio.

Echinospermum Virginicum, soldiers. E. Mass.

\section{CONVOLVULACEE.}

Convolvulus sepium, creepers. Mansfield, O.

Rutland beauty. Temple, N. H.

Cuscuta sp., love-vine. Fort Worth, Tex.

\section{SOLANACEAE.}

$\left.\begin{array}{l}\text { Datura Stramonium, } \\ \text { Datura Tatula, }\end{array}\right\}$ Jimson or Jimpson ${ }^{1}$ weed. W. and S. Lycium vulgare, privy; Jackson vine; jasmine. Mansfield, O. jessamine. Stratham, N. H.

\section{SCROPHULARIACEE.}

Linaria vulgaris, Jacob's ladder. Parts of N. E. bread-and-butter. Ipswich, Mass. dead men's bones. Troy, N. Y.

Antirrhinum majus, lion-mouth. Mansfield, O.

Chelone glabra, bammany (for balmony?). Belleisle, N. B.

Gerardia quercifolia (?), corn-flower. Hillsborough Co., N. H. pedicularia (?)

Castilleia coccinea, paint-brush. Peoria, Ill.; N. H. ; Hemmingford, P. Q.

Indian paint-brush. Mass.

Red Indians. Mass.

Wickakee. ${ }^{2}$ Mass.

election posies. Dudley, Mass.

prairie fire. Wisconsin.

1 Evidently a corruption of Jamestown, where the plant is most abundant. This corrupted form of the name is universal.

2 An Indian name. 


\section{BIGNONIACE E.}

Tecoma radicans, foxglove. Chestertown, Md.

VERBENACE $Æ$.

Verbena stricta, fever-weed. ${ }^{1}$ Peoria, Ill.

\section{LABIATÆ.}

Nepeta Glechoma, Robin runaway. N. H. creeping Charlie ; Jack-over-the-ground ; Gill-over. the-ground. E. Mass.

wild snake-root. Cambridge, Mass. crow-victuals. $^{2}$ Chestertown, $\mathrm{Md}$.

Brunella vulgaris, carpenter-weed. N. H.

NYCTAGINACEÆ.

Mirabilis Falapa, pretty-by-night. Fort Worth, Tex.

AMARANTACE E.

Gomphrena globosa, French clover. No. Ohio. globes. So. Vt.

\section{CHENOPODIACEÆ.}

Salicornia herbacea, chicken's toes. Kittery, Me.

\section{POLYGANACER.}

Rhoum Rhaponticum, pie-plant. General in Middle States and westward.

Rumex acetosella, horse-sorrel. Mansfield, O.

toad's sorrel. Stratham, N. H.

cow-sorrel. ${ }^{3}$ Miramichi, N. B.

gentlemen's sorrel. Cambridge, Mass.

sheep-sorrel. Wisconsin; So. Vt.

Polygonum aviculare, wire-grass. No. Ohio.

$$
\text { door-grass. So. Ind. }
$$

Polygonum acre, turkey-troop. Long Island, N. Y.

\section{EUPHORBIACEE.}

Euphorbia maculata, milkweed. No. Ohio.

Euphorbia marginata, Snow-on-the-mountains. N. H. ; Neb.

Euphorbia Cyparissias, tree-moss. Mansfield, O.

cypress. Rye Beach, N. H.

butternut. Harmony, Me.

Irish moss. N. B.

1 Thought to be a specific for fever and ague.

2 Name used by the negroes.

${ }^{8}$ Usually pronounced "cow-serls." 
Euphorbia Lathyris, mole-tree. ${ }^{1}$ No. Ohio.

JUGLANDACE $F$.

Carya tomentosa, bull-nut. Peoria, Ill.

MYRICACEE.

Myrica cerifera, candle-berry. Worcester Co., Mass.

CONIFERE.

Larix Americana, Juniper-tree. Newfoundland.

Funiperus communis, hackmatack. Ipswich, Mass.

fairy circle. E. Mass.

Funiperus sabina, var. procumbens, savin. ${ }^{2}$ Newfoundland.

ORCHIDACE $Æ$.

Arethusa bulbosa, dragon's mouth. Dudley, Mass.

Habenaria orbiculata, Solomon's seal. Barre, Vt.

Habenaria fimbriata, meadow pink. Mass.

Cypripedium acaule, nerve-root. N. B.

whip-poor-will. Boston, Mass.

Cypripedium spectabile, nerve-root. N. B.

whip-poor-will shoes. Conn.

SCITAMINEAE.

Canna Indica, adder's spear. Waltham, Mass.

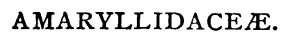

Narcissus Pseudo-Narcissus, Easter-flower. Mansfield, O. daffy. Stratham, N. H.

Narcissus poeticus, single daffy. Stratham, N. H.

IRIDACE E.

Iris pumila, crocus. Stratham, N. H.

Iris versicolor, poison flag.

$\left.\begin{array}{l}\text { flag-lily. } \\ \text { water-flag. } \\ \text { liver-lily. } \\ \text { snake-lily. }\end{array}\right\}$

Belamcanda Chinensis, dwarf tiger-lily. Mansfield, O.

1 Supposed to keep moles out of gardens.

2 The berries used in domestic medicine, and called face-and-eye berries.

8 These names are taken from Hobbs' Botanical Handbook. 
LILIACE $Æ$.

Smilax rotundifolia, biscuit-leaves ; bread-and-butter. ${ }^{1}$ Allston, Mass. wait-a-bit. $^{2}$ E. Mass.

nigger-head. Miramichi, N. B.

Muscari botryoides, baby's breath. E. Mass.

bluebell. Chestertown, Md.

bluebottle. Mansfield, O.

Yucca filamentosa, thread-and-needle. Mass.; N. Y.

Eve's darning needle. Fort Worth, Texas.

Maianthemum Canadense, cowslip. Dennysville, Me.

lily-of-the-valley; two-leaved Solomon's seal. N. H.

Clintonia borealis, cow-tongue. Aroostook Co., Me.; N. B.

$$
\text { heal-all. N. B. }
$$

Oakesia sessilifolia, wild oats. N. H.

Lilium superbum, nodding lilies; Turk's head. Mass.

Erythronium Americanum, yellow bells. Boston (?).

Trillium erectum, dish-cloth or stinking dish-cloth. Franklin Center, P. Q.

bumble-bee root. New England.

squaw-root. N. H.

Benjamins. So. Vt.

stinking Benjamin. N. B. (Any Trillium in N. B. is called Benjamin.)

Trillium grandiflorum, white lilies. No. Ohio ; Chestertown, Md.

Trinity lily. Wisconsin.

Trillium erythrocarpum, Benjamins. New England.

COMMELINACE

Tradescantia crassifolia, wandering Jew. General. inch-plant. Salem, Mass. joint-plant. Cambridge, Mass. Jacob's ladder. Hemmingford, P. Q.

ARACEÆ.

Ariscema triphyllum, bog onion. Worcester Co., Mass.

wild turnip. Stowe, Vt.

Jack-in-the-pulpit. General.

Symplocarpus fotidus, Polk-weed (poke weed ?). Brookline, Mass.

1 The young leaves eaten by children.

2 On account of the difficulty of tearing loose clothing caught by its stout prickles. 
GRAMINEÆE.

Cenchrus tribuloides, sand-burr. Ill. and westward.

Zea mays, a species of pop-corn, with variegated ears; guinea-corn. ${ }^{1}$ Mansfield, O.

yellow kernels, striped with red ; calico corn. Ill. long, indented kernels; dent corn. General. horse-tooth corn. Central Ill.

FILICES.

Pteris aquilina, hog-brake. N. H.

Osmunda regalis, buck-horn. Worcester Co., Mass.

Osmunda cinnamomea, fiddle-heads. ${ }^{2}$ Central Me.

Osmunda sp., fiddle-heads. Petit Codiac, N. B.

\section{LYCOPODIACEA.}

Lycopodium clavatum, coral evergreen. Stratham, N. H. creeping Jenny. N. B.

Lycopodium dendroideum, bunch evergreen. Stratham, N. H. crowfoot. Chestertown, Md.

Lycopodium complanatum, creeping Jenny. Bedford, Mass. liberty. Chestertown, Md. ground-cedar. N. B.

MUSCINEF.

Polytrichum commune, bears' bread. Dennysville, Me. rum-suckers. ${ }^{3}$ Stratham, N. H.

Bryum sp., robin-wheat. Mansfield, O.

FUNGI.

Hymenomycetes (any umbrella-shaped species), devil's umbrellas. Baltimore, Md.

Phallus sp., death-baby. ${ }^{4}$ Salem, Mass.

Ustilago Maydis (the smut of Indian corn), Devil's snuff-box. Chestertown, Md.

Cladonia bellidiflora (a common lichen), red-cup moss. General in N. E.

1 Because speckled like a guinea-fowl.

2 Under this name the unrolling fronds considerably sought and eaten as "greens."

3 So called from the supposed spirituous taste of the pasty mass of unripe spores.

- Name given from the fancy that they foretell death in the family near whose house they spring up. I have known of intelligent people rushing out in terror and beating down a colony of these as soon as they appeared in the yard.

vOL. v. - NO. I 7 . 
Usnea sp. (a tufted hair-like lichen), whisker-moss. Mansfield, O.

ALGÆ.

Laminaria (saccharina?). Venus's apron-strings. Brookline, Mass. Laminaria longicnuris, Devil's apron-strings ; Deb's apron-strings. Portland, Me.

Devil's apron. N. E. coast.

Spirogyra and allied confervaceae, frog-spit. U. S. frog-spawn. Parts of N. B

\section{BRIEFER ARTICLES.}

The systematic position of Entosthodon Bolanderi.--In February, I889, Dr. Edward Palmer, collecting in Lower California for the Department of Agriculture, found this species in thevicinity of Port San Quentin, about a hundred miles south of San Diego. This greatly extends southward the range of this species. It looks much like Funaria Californica Sulliv. \& Lesq., in outward appearance, but differs from it in the more acuminate leaves, in the capsule more long-necked and constricted under the orifice when dry and in the mamillate lid. Closer examination of the plants, which are in excellent condition, shows furthermore only a rudimentary peristome; the costa ceases above the middle of the leaf; the cells near the apex of the leaf are more elongated.

A search in the material of the closely allied genus Entosthodon led to the discovery of this identical species under the name of Entosthodon Bolanderi Lesq. The one specimen in the National Herbarium comes from the herbarium of Lesquereux himself, and is labelled: "Entosthodon Bolanderi Lesq. Ad terram argillosam, prope San Francisco, Californiæ. No. 236. Leg. Bolander."

A comparison of Palmer's plants with this specimen, and with the figures in Sulliv. Icon. Suppl. t. 7 , shows them to agree in all respects, except that the figure and description make no reference to the inner rudimentary peristome, distinctly present in the specimens of Lesquereux collected by Bolander, as well as in Palmer's specimens. This peristome is as pronounced as in Funaria microstoma. In specimens of Funaria Californica in the National Herbarium, it is not nearly so well developed as figured in Sulliv. Icon. Suppl. t. I8, but is almost as 